\title{
Influência do Sistema de Vulcanização nas Propriedades da Mistura NBR/EPDM
}

\author{
Marcia G. Oliveira, Bluma G. Soares \\ Instituto de Macromoléculas Professora Eloisa Mano, UFRJ
}

\begin{abstract}
Resumo: A mistura NBR/EPDM apresenta diferentes velocidades de cura, o que resulta na vulcanização heterogênea das fases, isto é, a fase NBR encontra-se em grande parte reticulada enquanto que a fase EPDM encontra-se pouco reticulada ou não-reticulada em casos extremos, prejudicando as propriedades finais da mistura. Este trabalho avalia os efeitos de diferentes sistemas de vulcanização à base de enxofre no processo de cura, na dureza, na resistência à tração, ao rasgamento e ao envelhecimento da mistura NBR/EPDM. Foram estudados os sistemas S/MBTS, S/TMTD e S/MBTS/TMTD variando-se a quantidade de enxofre em 0,3 e 1,0 phr. O processo de cura é influenciado pelo tipo de acelerador (MBTS, TMTD e a combinação destes) e pela quantidade de enxofre $(0,3$ e 1,0 phr) presente nas formulações. Esta influência é percebida na resistência à pré-cura e na velocidade de cura da mistura NBR/EPDM. A reticulação da fase EPDM também varia com o sistema de vulcanização empregado e influi diretamente nas propriedades mecânicas e na resistência ao envelhecimento.
\end{abstract}

Palavras-chave: Mistura de elastômeros, sistema de vulcanização, NBR, EPDM.

\section{The Influence of the Vulcanizing System on Curing, Mechanical and Ageing Properties of NBR/EPDM Blends}

Abstract: NBR and EPDM differ in the number of double bonds and also in polarity, thus presenting different cure rates. Their blends frequently result in a maldistribution of crosslinks. In this work, sulfur-based systems varying in accelerator type and sulfur amount were investigated as vulcanizing systems for NBR/EPDM blends. The accelerator type and the sulfur concentration affected the curing characteristics of the blends. Some formulations were able to crosslink the EPDM phase to a larger extent and consequently displayed better mechanical and ageing properties.

Keywords: Curing system, elastomer blends, NBR, EPDM.

\section{Introdução}

Misturas de dois ou mais elastômeros visam atingir objetivos específicos, como a melhoria das propriedades físicas, da resistência à ação do calor, oxigênio e ozônio bem como das características de processamento. A borracha nitrílica (NBR) possui excelente resistência a óleo e abrasão. Sua mistura com borrachas com baixo índice de insaturação, como o terpolímero de etileno-propileno-dieno (EPDM) ou a borracha butílica (BR), é um modo atrativo de desenvolver novos materiais mais resistentes à ação do calor, oxigênio e ozônio. Entretanto, a literatura ${ }^{[1-4]}$ relata que as propriedades físicas destas misturas são geralmente inferiores àquelas previstas a partir das propriedades de cada um dos elastômeros, principalmente devido às diferenças de polaridade e teor de insaturação dos componentes. A diferença no teor de insaturação resulta em diferentes reatividades quanto à vulcanização com enxofre, isto é, diferentes velocidades de cura e densidade de ligações cruzadas. A diferença de polaridade resulta numa solubilidade maior do enxofre e dos aceleradores orgânicos no elastômero mais insaturado e polar. Grande parte dos agentes de cura são, então, consumidos pelo elastômero mais insaturado (NBR), resultando numa

Autor para correspondência: Bluma G. Soares, Instituto de Macromoléculas Professora Eloisa Mano, UFRJ, Centro de Tecnologia, Bloco J, Ilha do Fundão, CEP:21945-970, Rio de Janeiro, RJ. E-mail: bluma@ima.ufrj.br 
cura incompleta do outro elastômero (EPDM por exemplo).

As indústrias de borracha normalmente utilizam processos de vulcanização empregando sistemas de vulcanização à base de enxofre. Atualmente, há uma vasta gama de aceleradores disponíveis no mercado que oferecem uma ampla faixa de escolha de velocidade de cura, tempo de pré-cura e propriedades finais. As classes de aceleradores mais importantes incluem as sulfenamidas, mercaptobenzotiazóis, tiurans e aminas. As formulações de borracha podem conter um ou a combinação de dois tipos de aceleradores. Freqüentemente, são utilizadas combinações de aceleradores. Quando combinados em proporções adequadas, sistemas binários podem melhorar significativamente o processo de cura e as propriedades mecânicas. Normalmente utilizam-se aceleradores da classe dos benzotiazóis combinados com aceleradores da classe dos tiurans ou aminas em pequenas quantidades. A escolha dos aceleradores (tipo e combinação) depende de alguns fatores como a natureza do elastômero, as propriedades finais e as condições de processamento $^{[5-8]}$.

A norma ASTM D-2934 recomenda para formulações de NBR o uso de sistema binário constituído por dissulfeto de tetrametiltiuram (TMTD) e n-tertbutil-2-benzotiazol-sulfenamida (TBBS). A combinação de benzotiazóis ou sulfenamidas com tiurans normalmente melhora a resistência à pré-cura, mantendo alta velocidade de cura, promovida pelos aceleradores do tipo tiuram ${ }^{[7]}$. Além deste sistema, a literatura relata outras formulações para misturas de elastômeros contendo NBR que também utilizam este mesmo tipo de combinação ou apenas aceleradores da classe dos benzotiazóis ${ }^{[9-11]}$. Em relação ao EPDM, a grande maioria dos estudos utiliza sistemas binários, os quais obrigatoriamente contêm aceleradores da classe dos tiurans como um dos componentes ${ }^{[10-13]}$.

Alguns sistemas de vulcanização para a mistura NBR/EPDM já foram estudados e incluem o uso de aceleradores do tipo tiuram com alto grau de alquilação ${ }^{[14]}$ e o uso de óxido de chumbo em substituição ao óxido de zinco ${ }^{[15]}$. Entretanto, estes aceleradores não se encontram comercialmente disponíveis hoje em dia. Além disso, do ponto de vista ambiental também não é recomendável o uso de óxido de chumbo. Também existem estudos que empregam a vulcanização com peróxidos, que apresenta a vantagem de uma distribuição uniforme deste entre os elastômeros componentes da mistura ${ }^{[16,17]}$. Mas este tipo de cura não é recomendável para várias aplicações em razão das inferiores propriedades dinâmicas do vulcanizado final. Embora, há alguns anos a mistura NBR/EPDM já seja objeto de estudo de alguns pesquisadores, ainda há falta de informação a respeito do que seria um sistema de vulcanização a base de enxofre mais adequado para esta mistura.

O presente trabalho investiga a influência do sistema de vulcanização no processo de cura e nas propriedades mecânicas da mistura NBR/EPDM, buscando estabelecer qual seria o melhor sistema de vulcanização capaz de vulcanizar ambas as fases. Para tanto, foram escolhidos o 2,2-ditiomercaptobenzotiazol (MBTS) e dissulfeto de tetrametiltiuram (TMTD) como aceleradores em sistemas de vulcanização simples ou ainda combinados em sistemas binários para a mistura NBR/ EPDM com composição igual a 70/30. O efeito da razão acelerador / enxofre também foi investigado.

\section{Experimental}

\section{Materiais}

Borracha nitrílica (NBR) com viscosidade mooney $\left(\mathrm{ML}_{1+4} @ 100^{\circ} \mathrm{C}\right)$ igual a 48 e razão acrilonitrila/butadieno igual a 33/67 \% (p/p), foi gentilmente cedida pela Nitriflex S.A.

Terpolímero de etileno-propileno-dieno (EPDM) contendo $60 \%$ de etileno e $1,14 \mathrm{mmol}$ de etileno norboneno/g de EPDM, foi gentilmente cedido pela DSM elastômeros S.A.

Óxido de zinco, ácido esteárico, enxofre, 2,2-ditiomercaptobenzotiazol (MBTS) e dissulfeto de tetrametiltiuram (TMTD), todos de grau comercial, foram usados como recebidos.

\section{Processo de Mistura}

As misturas NBR/EPDM na composição 70/30 foram preparadas em misturador de cilindros a $110^{\circ} \mathrm{C}$. Primeiro a NBR foi mastigada por 3 minutos, e então adicionou-se o EPDM. Em seguida a cada 2 minutos foram adicionados o óxido de zinco, o ácido esteárico, o enxofre e finalmente os aceleradores (MBTS e/ou TMTD).

\section{Determinação dos Parâmetros de Vulcanização}

As misturas cruas foram analisadas em reômetro de disco oscilatório a $160^{\circ} \mathrm{C}$ com arco $1^{\circ}$, segundo a norma 
ASTM D-2084-81. A partir dos reogramas foram determinados os valores de torque máximo $(\mathrm{MH})$, torque mínimo (ML), tempo de pré-cura $\left(\mathrm{t}_{\mathrm{s}} 1\right)$ e tempo ótimo de cura $\left(\mathrm{t}_{90}\right)$. As misturas foram vulcanizadas em prensa hidráulica aquecida a $160^{\circ} \mathrm{C}$ no tempo ótimo de cura.

\section{Ensaios de Inchamento}

Corpos de prova retangulares de $20 \times 10 \times 2 \mathrm{~mm}$, previamente pesados, foram imersos em tolueno e em metil-etil-cetona (MEK) por sete dias. A cada 24 horas os solventes foram trocados para evitar a saturação dos mesmos. Ao término do ensaio, os corpos de prova foram pesados e depois secos a vácuo e novamente pesados. $\mathrm{O}$ volume de borracha presente na rede inchada (Vr) foi calculado conforme a Equação $1^{[18]}$.

$$
V_{r}=\frac{M_{f} \times \rho_{r}^{-1}}{M_{f} \times \rho_{r}^{-1}+\left(M_{i}-M_{f}\right) \times \rho_{s}^{-1}}
$$

Onde: $\mathrm{M}_{\mathrm{f}}$ é a massa seca após o inchamento, $\rho_{\mathrm{r}}$ é a densidade da mistura, $M_{i}$ é a massa inchada e $\rho_{\mathrm{s}}$ é a densidade do solvente (tolueno $-0,867 \mathrm{~g} \mathrm{x} \mathrm{cm}^{-3} \mathrm{e}$ MEK - 0,8054 $\mathrm{g} \mathrm{x} \mathrm{cm}^{-3}$ ).

\section{Propriedades Mecânicas}

A dureza foi determinada em durômetro do tipo Shore A, segundo a norma ASTM D-2240-86. Os ensaios de compressão foram realizados em estufa de circulação de ar a $70^{\circ} \mathrm{C}$ por 22 horas, seguindo o método B da norma ASTM D-395-69. A resistência ao rasgamento foi determinada segundo a norma
ASTM D. Os ensaios de resistência à tração seguiram a norma DIN 53504. Os ensaios de envelhecimento foram realizados em estufa com circulação de ar a $70^{\circ} \mathrm{C}$ por 72 horas, segundo norma ASTM D-573-81.

\section{Ensaios Dinâmico-M ecânicos}

As análises dinâmico-mecânicas foram realizadas em DMTA (Rheometric Scientific MKIII) no modo flexão na frequência de $1 \mathrm{~Hz}$. A faixa de temperatura estudada foi de $-60^{\circ} \mathrm{C}$ até $20^{\circ} \mathrm{C}$ com velocidade de aquecimento de $2^{\circ} \mathrm{C} / \mathrm{min}$.

\section{Resultados e Discussão}

\section{Parâmetros de Vulcanização}

Os parâmetros de vulcanização encontram-se na Tabela 1. O sistema contendo apenas MBTS como acelerador apresenta maiores tempos de cura e resistência à pré-cura do que os sistemas nos quais o acelerador é o TMTD. Este comportamento combina com a classificação do MBTS como um semi-ultra-acelerador de ação retardada enquanto que o TMTD é classificado como ultra-acelerador e também atua como agente de vulcanização. A combinação de dois aceleradores é empregada quando se deseja alcançar uma maior segurança de processamento ${ }^{[5-7]}$. O sistema binário MBTS/TMTD apresenta melhor resistência a précura quando comparado ao sistema contendo apenas TMTD, entretanto os tempos ótimos de cura são similares. O aumento da quantidade de enxofre nas formulações resulta em menores tempos de cura e pré-cura.

Tabela 1. Formulações e parâmetros de vulcanização das misturas NBR/EPDM (70/30)

\begin{tabular}{|c|c|c|c|c|c|c|c|}
\hline \multicolumn{3}{|c|}{ Formulações ${ }^{\mathrm{a}}$} & \multicolumn{5}{|c|}{ Parâmetros de Vulcanização } \\
\hline $\begin{array}{c}\mathrm{S} \\
(\mathrm{phr})\end{array}$ & $\begin{array}{c}\text { MBTS } \\
\text { (phr) }\end{array}$ & $\begin{array}{c}\text { TMTD } \\
\text { (phr) }\end{array}$ & $\begin{array}{c}\mathrm{Ml}^{\mathrm{b}} \\
\text { (lb.in) }\end{array}$ & $\begin{array}{c}\mathrm{Mh}^{\mathrm{c}} \\
\text { (lb.in) }\end{array}$ & $\begin{array}{l}\mathrm{ts} 1^{\mathrm{d}} \\
(\mathrm{min})\end{array}$ & $\begin{array}{l}\mathrm{t} 90^{\mathrm{e}} \\
(\mathrm{min})\end{array}$ & $\begin{array}{c}\mathrm{k} @ 160^{\circ} \mathrm{C}^{\mathrm{f}} \\
\left(\min ^{-1}\right)\end{array}$ \\
\hline 0,3 & 2,0 & - & 4,0 & 14,6 & 21,6 & 43,2 & 0,0843 \\
\hline 1,0 & 2,0 & - & 3,6 & 23,0 & 12,0 & 25,2 & 0,2107 \\
\hline 0,3 & - & 1,0 & 3,9 & 15,1 & 3,1 & 7,2 & 0,2930 \\
\hline 1,0 & - & 1,0 & 4,4 & 19,6 & 2,9 & 5,6 & 0,5272 \\
\hline 0,3 & 2,0 & 1,0 & 3,7 & 18,0 & 4,9 & 8,3 & 0,4791 \\
\hline 1,0 & 2,0 & 1,0 & 3,6 & 24,4 & 3,5 & 6,1 & 0,8782 \\
\hline
\end{tabular}

a Todas as formulações contêm 5,0 phr de óxido de zinco e 0,5 phr de ácido esteárico

b Torque mínimo

c Torque máximo

d Tempo de pré-cura

e Tempo ótimo de cura

${ }^{\mathrm{f}}$ Constante cinética de vulcanização à $160^{\circ} \mathrm{C}$ 
De acordo com a literatura, um aumento na quantidade de enxofre torna a cura mais rápida e este fenômeno é ainda dependente da razão acelerador/enxofre ${ }^{[19]}$.

Os sistemas simples (S/MBTS e S/TMTD) com uma quantidade menor de enxofre produzem valores semelhantes de Мн. Os valores de torque máximo, medidos à pequenas deformações, são normalmente proporcionais à quantidade de ligações cruzadas formadas por volume de borracha, isto é, à densidade de ligações cruzadas. Estes sistemas, caracterizados por uma quantidade de acelerador maior do que a quantidade de enxofre, são normalmente classificados como sistemas eficientes e sua principal característica é a formação de ligações mono e dissulfídicas em grande quantidade. $\mathrm{O}$ aumento na quantidade de enxofre nestes sistemas promove um aumento também na quantidade de ligações polissulfídicas e no grau de reticulação, como é indicado pelos valores de Мн. Neste caso, dentre os sistemas simples, os maiores valores de MH são encontrados quando o acelerador é o MBTS. O sistema binário é o que apresenta os maiores valores de $\mathrm{MH}$ em ambas as razões acelerador/enxofre estudadas.

\section{Parâmetros cinéticos de vulcanização}

A equação cinética que descreve o processo de vulcanização pode ser obtida através dos valores de torque e tempo dos reogramas gerados nos ensaios em reômetro de disco oscilatório. Se o processo for considerado como uma reação de primeira ordem, este pode ser expresso como:

$$
\ln (\mathrm{MH}-\mathrm{Mt})=\mathrm{kx} \mathrm{t}+\ln \left(\mathrm{MH}_{\mathrm{H}}-\mathrm{ML}\right)
$$

Onde ML, Mt, Mн e k são, respectivamente, torque mínimo, torque no tempo de cura t, torque máximo e constante cinética de vulcanização ${ }^{[20-24]}$. Construindo-se o gráfico do ln (MH - Mt) contra o tempo, observa-se uma relação linear como é mostrado na Figura 1, indicando que a vulcanização da mistura NBR/EPDM segue uma cinética de primeira ordem. Os valores da constante cinética de vulcanização (k) foram obtidos através do coeficiente angular das retas e encontram-se na Tabela 1. Estes valores aumentam de acordo com o aumento da quantidade de enxofre, confirmando o comportamento já observado para o tempo de cura, ou seja o aumento da quantidade de enxofre torna a vulcanização mais rápida. Como era esperado, os valores da constante cinética de vulcanização aumentam na seguinte ordem:

MBTS < TMTD < MBTS/TMTD
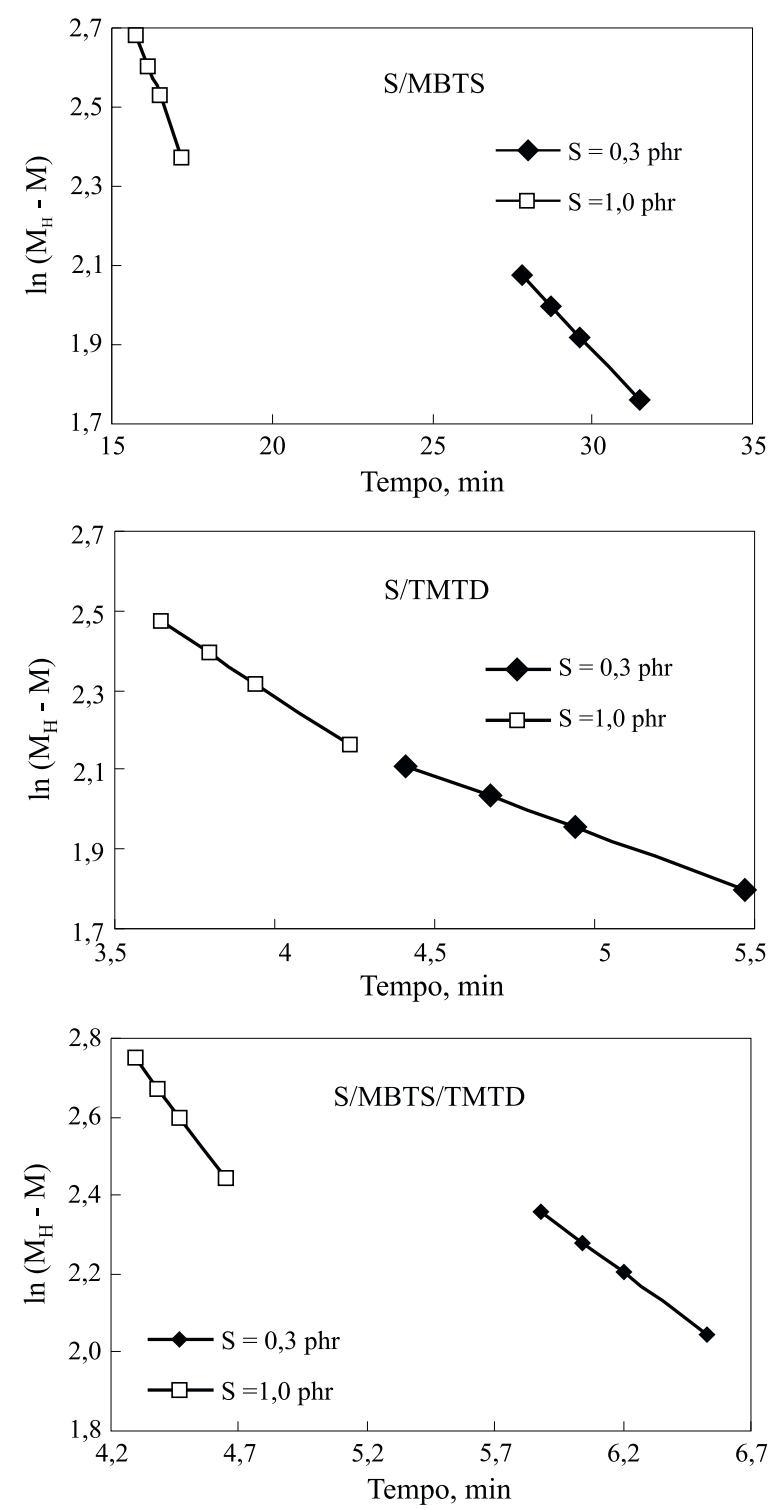

Figura 1. Gráfico do $\ln \left(\mathrm{M}_{\mathrm{H}}-\mathrm{M}\right)$ contra o tempo para as misturas NBR/ EPDM (70/30)

\section{Densidade de Ligações Cruzadas}

A densidade de ligações cruzadas pode ser estimada a partir dos ensaios de inchamento em solvente, medindo-se a fração em volume de borracha presente na rede inchada $(\mathrm{Vr})$, calculada pela Equação 1. Os ensaios de inchamento foram feitos em MEK (metiletil cetona) e em tolueno. A MEK é capaz de inchar e dissolver apenas a fase NBR enquanto que o tolueno é capaz de inchar e dissolver tanto a fase NBR como a fase EPDM. A Tabela 2 apresenta os valores obtidos para Vr e também a quantidade de material nãoreticulado (material solúvel) para as formulações estudadas. Os valores de Vrobtidos pelo inchamento em 
Oiveira, M. G.; Soares, B. G. - Influência dos sistema de vulcanização nas propriedades da mistura NBR/EPDM

Tabela 2. Resultados dos ensaios de inchamento das misturas NBR/EPDM (70/30)

\begin{tabular}{ccccccc}
\hline & $\begin{array}{c}\text { Sistema de vulcanização } \\
(\mathbf{p h r})\end{array}$ & & \multicolumn{2}{c}{$\begin{array}{c}\text { Volume de borracha } \\
\text { presente na rede inchada } \\
(\mathbf{V r})\end{array}$} & \multicolumn{2}{c}{$\begin{array}{c}\text { Material solúvel } \\
(\%)\end{array}$} \\
\hline S & MBTS & TMTD & MEK & tolueno & MEK & tolueno \\
\hline 0,3 & 2,0 & - & 0,1732 & 0,1255 & 3,8 & 9,5 \\
1,0 & 2,0 & - & 0,2864 & 0,2119 & 3,1 & 1,7 \\
0,3 & - & 1,0 & 0,2338 & 0,2134 & 3,6 & 30,0 \\
1,0 & - & 1,0 & 0,2904 & 0,2023 & 2,2 & 13,1 \\
0,3 & 2,0 & 1,0 & 0,2687 & 0,1925 & 3,4 & 30,0 \\
1,0 & 2,0 & 1,0 & 0,3442 & 0,2329 & 3,1 & 10,0 \\
\hline
\end{tabular}

MEK são sempre maiores, visto que este solvente não é capaz de inchar a fase EPDM. A fase EPDM nãoinchada pode limitar o inchamento da matriz de NBR do mesmo modo como o negro de fumo restringe o inchamento de uma matriz elastomérica ${ }^{[25,26]}$. A quantidade não-reticulada de fase NBR é muito pequena para todos os sistemas estudados, como é indicado pela quantidade de material solúvel obtido após extração com MEK. O grau de reticulação da fase NBR (obtido por inchamento em MEK) aumenta na seguinte ordem: MBTS < TMTD < MBTS/TMTD

Aumentado-se a quantidade de enxofre nas formulações, aumenta-se também o grau de reticulação, o que combina com os valores de torque obtidos nos experimentos em reômetro de disco oscilatório.

Os experimentos feitos em tolueno fornecem menores valores de $\mathrm{Vr}$, uma vez que este solvente é capaz de inchar ou dissolver ambas as fases. Para as misturas vulcanizadas com menor quantidade de enxofre $(0,3$ phr), o grau de reticulação é maior para as formulações contendo TMTD como acelerador, sozinho ou combinado com MBTS. É importante observar que a quantidade de material solúvel nas misturas vulcanizadas apenas com MBTS é significativamente menor que nos outros sistemas. Considerando que a quantidade de EPDM nas misturas corresponde a 30\% e que a quantidade de material solúvel na mistura vulcanizada com o sistema S/MBTS (0,3:2,0 phr) corresponde a 7-8\%, pode-se concluir que grande parte do EPDM presente na mistura foi vulcanizada. Apesar do alto grau de reticulação observado para as misturas vulcanizadas com o sistemas S/TMTD (0,3:1,0 phr) e S/MBTS/TMTD (0,3:2,0:1,0 phr), a quantidade de material solúvel é bem alta, indicando uma pequena participação do EPDM na rede de liga- ções cruzadas. O aumento da quantidade de enxofre nas formulações reduz a quantidade de material solúvel, sugerindo que a fase EPDM tenha sido vulcanizada. Novamente, o sistema S/MBTS é capaz de vulcanizar a fase EPDM numa extensão maior.

Os valores de torque obtidos no reômetro de disco oscilatório são similares para os sistemas S/MBTS (0,3:2,0 phr) e S/TMTD (0,3:1,0 phr) enquanto que o grau de reticulação é bem maior para o segundo sistema. Comparando-se estes dois sistemas empregados em formulações com maior quantidade de enxofre, os graus de reticulação são bastante semelhantes, embora o sistema S/MBTS tenha apresentado maiores valores de torque. Estes valores relativamente maiores de torque para o sistema S/MBTS (1,0:2,0 phr) podem ser atribuídos a uma quantidade maior da fase EPDM presente na rede de ligações cruzadas.

\section{Propriedades Mecânicas}

Os resultados de dureza, resistência à tração, ao rasgamento e à compressão são apresentados na Tabela 3 .

A dureza é proporcional à quantidade de ligações cruzadas formadas durante o processo de vulcanização, sendo assim é diretamente afetada pela quantidade de enxofre presente no sistema de vulcanização. Quanto maior for a quantidade de enxofre maior será a dureza e maior será o grau de reticulação. Este comportamento é justamente o que foi obtido. Os sistemas de vulcanização contendo $1,0 \mathrm{phr}$ de enxofre apresentaram valores de dureza ligeiramente maiores, indicando um maior grau de reticulação destas misturas. Dentre os sistemas estudados, os que contêm TMTD e 1,0 phr de enxofre foram os que alcançaram maior dureza. 
Oiveira, M. G; Soares, B. G. - Influência dos sistema de vulcanização nas propriedades da mistura NBR/EPDM

Tabela 3. Propriedades mecânicas da mistura NBR/EPDM (70/30)

\begin{tabular}{|c|c|c|c|c|c|c|}
\hline & \multicolumn{6}{|c|}{ Sistemas de Vulcanização } \\
\hline & \multicolumn{2}{|c|}{ S/MBTS } & \multicolumn{2}{|c|}{ S/TMTD } & \multicolumn{2}{|c|}{ S/MBTS/TMTD } \\
\hline & $\mathbf{0 , 3 / 2 , 0}$ & $1,0 / 2,0$ & $\mathbf{0 , 3 / 1 , 0}$ & $1,0 / 1,0$ & $0,3 / 2,0 / 1,0$ & $1,0 / 2,0 / 1,0$ \\
\hline Dureza, Shore A & $41(1)$ & $43(1)$ & $44(1)$ & $47(1)$ & $42(1)$ & $48(1)$ \\
\hline $\mathrm{DPC}^{\mathrm{a}}, \%$ & 58,2 & 48,1 & 57,9 & 57,0 & 41,6 & 34,7 \\
\hline Rasgamento, N/m & $10,9(0,1)$ & $11,0(0,1)$ & $13,1(0,1)$ & $13,0(0,1)$ & $10,3(0,1)$ & $10,9(0,1)$ \\
\hline$M_{300}^{b}$ & $0,8(0,0)$ & $1,4(0,1)$ & $1,2(0,1)$ & $1,6(0,1)$ & $1,3(0,1)$ & $2,7(0,2)$ \\
\hline$\sigma^{\mathrm{c}}, \mathrm{Mpa}$ & $3,0(0,3)$ & $2,7(0,2)$ & $2,6(0,2)$ & $2,5(0,1)$ & $2,2(0,2)$ & $3,0(0,3)$ \\
\hline Retenção da $\sigma, \%$ & 85 & 95 & 88 & 92 & 95 & 97 \\
\hline$\varepsilon^{\mathrm{d}}, \%$ & $1000(90)$ & $600(20)$ & $560(40)$ & $470(30)$ & $570(20)$ & $320(20)$ \\
\hline Retenção da $\varepsilon, \%$ & 83 & 95 & 99 & 89 & 95 & 105 \\
\hline
\end{tabular}

Os valores de tensão na ruptura não são substancialmente influenciados pelo sistema de vulcanização quando MBTS ou TMTD são empregados como aceleradores. Valores ligeiramente melhores de tensão na ruptura foram observados para o MBTS. O aumento da quantidade de enxofre nestes dois sistemas resulta numa pequena redução dos valores de tensão na ruptura, apesar de aumentar o grau de reticulação. Com relação ao sistema binário, o menor valor de tensão na ruptura é obtido quando a menor quantidade de enxofre é empregada. Este valor aumenta razoavelmente quando a quantidade de enxofre aumenta.

A deformação na ruptura diminui de acordo com o aumento da quantidade de enxofre, provavelmente devido a uma maior densidade de ligações cruzadas e também a uma quantidade maior de fase EPDM que se encontra reticulada. Conforme pode ser observado na Tabela 2 , há uma redução na quantidade de material solúvel formado durante os ensaios de inchamento em tolueno para as misturas vulcanizadas com sistemas contendo $1,0 \mathrm{phr}$ de enxofre, sinalizando a melhoria da reticulação da fase EPDM. O maior valor de deformação na ruptura foi obtido empregando-se MBTS como acelerador e uma pequena quantidade de enxofre ( $0,3 \mathrm{phr})$.

A resistência ao rasgamento não é significativamente afetada pela quantidade de enxofre para um mesmo sistema de vulcanização. Em relação aos diferentes sistemas de vulcanização, o sistema simples
S/TMTD é o que apresenta melhor resistência ao rasgamento.

Todos os sistemas de vulcanização aumentam a resistência à compressão quando é usada uma quantidade maior de enxofre ( $1,0 \mathrm{phr})$. Esta tendência pode ser explicada pelo aumento da densidade de ligações cruzadas tornando maior a rigidez do vulcanizado, conforme pode ser visto pelo valor do módulo a $300 \%$ de deformação $\left(\mathrm{M}_{300}\right)$, e assim reduzindo a sua capacidade de deformação durante a compressão. A quantidade de material solúvel formado durante o ensaio de inchamento corrobora os resultados da resistência à compressão, isto é a redução da quantidade de material solúvel é acompanhada por um aumento da resistência à compressão. É interessante notar que a mistura vulcanizada com o sistema S/MBTS/TMTD $(1,0 / 2,0 / 1,0)$ apresenta a maior resistência à compressão, embora não seja a que apresente a menor quantidade de material solúvel. Porém, a resistência à compressão assim como outras propriedades mecânicas não é função apenas da densidade de ligações cruzadas, mas também do tipo de ligação formada. A característica deste sistema, em particular, é a formação de ligações mono e dissulfídicas, as quais são mais resistentes à compressão e à tração, mas em compensação a deformação do vulcanizado nos testes de resistência à tração é reduzida.

A estabilidade térmica das misturas NBR/EPDM foi investigada, submetendo-as a um processo de en- 
velhecimento em estufa de circulação de ar a $70^{\circ} \mathrm{C}$ por 72 horas, após o qual foi analisada a resistência à tração. A Tabela 4 apresenta os resultados referentes à retenção dos valores de tensão e deformação na ruptura após o envelhecimento. É interessante notar que todos os sistemas contendo uma quantidade maior de enxofre apresentaram uma retenção maior dos valores de tensão na ruptura. Este comportamento difere do normalmente relatado na literatura, segundo o qual um aumento na quantidade de ligações polissulfídicas, resultante do aumento da quantidade de enxofre, reduz a estabilidade térmica do vulcanizado $^{[5,7]}$. Este comportamento inesperado neste estudo pode ser atribuído a um maior grau de reticulação da fase EPDM obtido com uma quantidade mais alta de enxofre. A fase EPDM participando da rede de ligações cruzadas pode proteger as ligações duplas remanescentes na fase NBR, desta forma reduzindo ou evitando a degradação.

\section{Propriedades Mecânico-Dinâmicas}

A Figura 2 ilustra a dependência do fator de amortecimento $(\tan \delta)$ com a temperatura para as misturas NBR/EPDM. As temperaturas de transição vítrea (Tg) referentes a cada uma das fases foram medidas nos pontos máximos das curvas do fator de amortecimento em função da temperatura e encontram-se na Tabela 4. Todas as misturas apresentaram duas transições vítreas correspondentes às fases EPDM e NBR, confirmando a incompatibilidade destes elastômeros.

Os baixos valores para o fator de amortecimento da fase EPDM estão relacionados diretamente com a menor quantidade desta fase presente na mistura (30\%). Os sistemas de vulcanização afetam diretamente a transição vítrea da fase NBR. Como é mostrado na Tabela 4, os sistemas S/MBTS e S/MBTS/TMTD têm a sua temperatura de transição vítrea referente a fase NBR deslocada para valores mais altos quando uma quantidade maior de enxofre é empregada. Este comportamento indica uma redução da mobilidade das cadeias da fase NBR como resultado do aumento do grau de reticulação desta fase, e também está de acordo com os resultados obtidos nos ensaios em reômetro de disco oscilatório e inchamento.

A variação na quantidade de enxofre no sistema S/TMTD não produz modificações nos valores de Tg de ambas as fases. Os valores de Vr obtidos a partir dos ensaios de inchamento também não apresentaram variações. Ambos os resultados revelam que a
S/MBTS
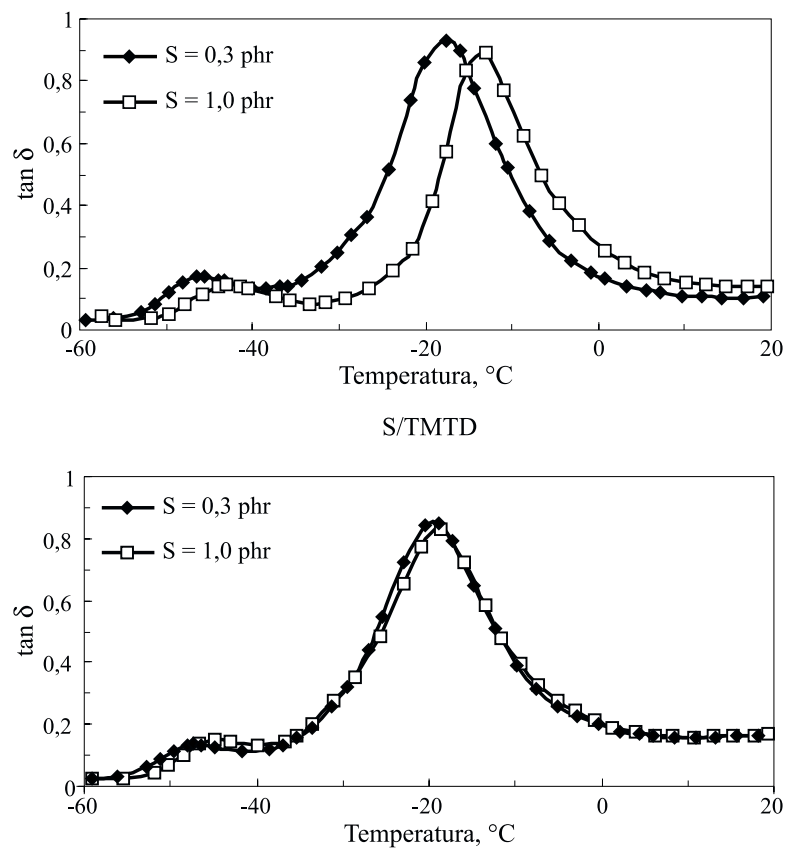

S/MBTS/TMTD

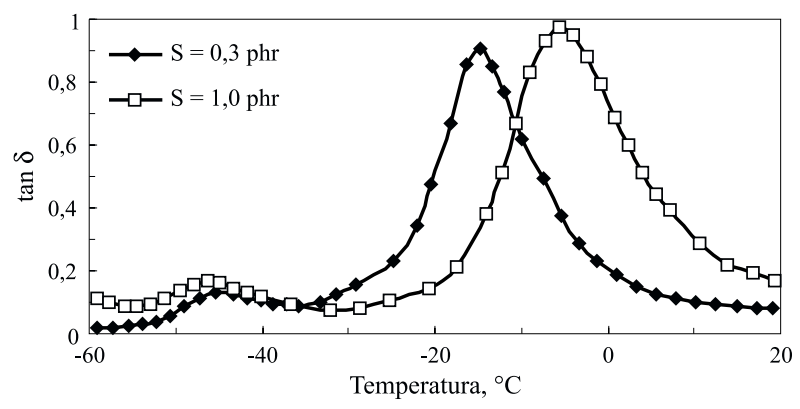

Figura 2. Curva do fator de amortecimento $(\tan \delta)$ contra a temperatura para as misturas NBR/EPDM (70/30)

concentração de enxofre na formulação não afeta o grau de reticulação das misturas vulcanizadas com o sistema S/TMTD.

Embora as misturas vulcanizadas com o sistema binário e o sistema S/MBTS (1,0:2,0 phr) tenham apresentado valores de $\mathrm{Vr}$ semelhantes aos valores de $\mathrm{Vr}$ das misturas vulcanizadas com o sistema S/TMTD, os valores de Tg para a fase NBR obtidos pelos ensaios de DMTA foram significativamente maiores. Estudos envolvendo as propriedades dinâmico-mecânicas da borracha natural vulcanizada sugerem que mudanças significativas na $\mathrm{Tg}$ podem ser decorrência da formação de grupos heterocíclicos ao longo da cadeia ${ }^{[27,28]}$. Do mesmo modo, as diferenças nos valores de Tg encontradas neste estudo não devem ser atribuídas apenas às diferenças no grau de reticulação mas também a outras interações de cunho intra ou intermolecular, 
Oiveira, M. G; Soares, B. G - Influência dos sistema de vulcanização nas propriedades da mistura NBR/EPDM

Tabela 4. Propriedades dinâmino-mecânicas da mistura NBR/EPDM (70/30)

\begin{tabular}{ccccccc}
\hline $\mathbf{S}$ & MBTS & TMTD & \multicolumn{2}{c}{ Fase EPDM } & \multicolumn{2}{c}{ Fase NBR } \\
\cline { 4 - 7 } $\mathbf{p h r}$ & $\mathbf{p h r}$ & $\mathbf{p h r}$ & $\mathbf{T g},{ }^{\circ} \mathbf{C}$ & $\mathbf{t a n} \boldsymbol{T}$ & $\mathbf{T g},{ }^{\circ} \mathbf{C}$ & $\mathbf{t a n} \boldsymbol{\delta}$ \\
\hline 0,3 & 2,0 & - & $-45,6$ & 0,1712 & $-17,6$ & 0,9290 \\
1,0 & 2,0 & - & $-43,1$ & 0,1438 & $-13,0$ & 0,8912 \\
0,3 & - & 1,0 & $-47,3$ & 0,1356 & $-18,9$ & 0,8520 \\
1,0 & - & 1,0 & $-44,9$ & 0,1498 & $-18,6$ & 0,8301 \\
0,3 & 2,0 & 1,0 & $-45,3$ & 0,1286 & $-14,6$ & 0,9042 \\
1,0 & 2,0 & 1,0 & $-46,4$ & 0,1661 & $-5,7$ & 0,9749 \\
\hline
\end{tabular}

que podem variar de acordo com o sistema de vulcanização empregado. Tais interações, incluindo a formação de grupos heterocíclicos ao longo da cadeia, podem ser responsáveis pelo aumento substancial dos valores de Tg com o aumento da quantidade de enxofre nas formulações para o sistema binário.

\section{Conclusões}

A influência do sistema de vulcanização nos parâmetros de cura, no grau de reticulação, nas propriedades mecânicas e na resistência ao envelhecimento das misturas NBR/EPDM foi investigada. O sistema de vulcanização composto por S/MBTS apresentou a melhor resistência à pré-cura enquanto que os sistemas de vulcanização contendo TMTD, simples ou binário, apresentaram processos de cura mais rápidos. As formulações mais ricas em enxofre apresentaram maiores valores para a diferença entre torques máximo e mínimo, que podem ser interpretados como um aumento no grau de reticulação. Esta tendência também foi observada para os valores de dureza e resistência à compressão.

O sistema de vulcanização S/MBTS apresentou melhor resistência à tração. De acordo com os testes de inchamento, este sistema é capaz de vulcanizar tanto a fase NBR como a fase EPDM, embora o grau de reticulação no geral não tenha sido alto. A maior reticulação da fase EPDM alcançada por este sistema pode ser responsável pelo melhor desempenho mecânico observado para estas formulações.

Com relação à resistência ao envelhecimento, o aumento da quantidade de enxofre em todas as formulações estudadas resultou em uma estabilidade térmica maior, a qual foi atribuída ao aumento do grau de reticulação da fase EPDM.
A Tg das formulações S/MBTS e S/MBTS/TMTD foram mais altas para as formulações mais ricas em enxofre, como conseqüência não só do aumento do grau de reticulação mas também da formação de grupos heterocíclicos ao longo da cadeia elastomérica. Este fenômeno contribui para uma redução da mobilidade molecular da cadeia polimérica. Os menores valores de $\mathrm{Tg}$ foram observados para as formulações S/TMTD. Além disso, para este sistema em particular, a quantidade de enxofre não afeta a $\mathrm{Tg}$.

Finalmente, a partir dos resultados apresentados neste trabalho, é possível concluir que o melhor sistema de vulcanização para a mistura NBR/EPDM deve ser o sistema simples S/MBTS em função da melhor performance mecânica associada à boa resistência ao envelhecimento apresentada por esta formulação.

\section{Agradecimentos}

Os autores agradecem ao CNPq, PADCT-CNPq (Proc. $\mathrm{n}^{\mathrm{o}}$ 620132/98-1), CEPG-UFRJ e CAPES pelo apoio financeiro.

\section{Referências Bibliográficas}

1. Duin, M.; Krans J. C. J. \& Smedinga, J. - Kauts. Gummi Kunsts., 46, p.445 (1993).

2. Coran, A.Y. - Rubber Chem. Technol., 61, p.281 (1988).

3. Gardiner, J. B. - Rubber Chem. Technol., 42, p.1058 (1969).

4. Shershnev, V. A. - Rubber Chem. Technol., 55, p.537 (1982).

5. Akiba, M. \& Hashim, A. S. - Prog. Polym. Sci., 22, p.475 (1997).

Polímeros: Ciência e Tecnologia, vol. 12, № 1, p. 11-19, 2002 
Oiveira, M. G.; Soares, B. G. - Influência dos sistema de vulcanização nas propriedades da mistura NBR/EPDM

6. Coran, A.Y. - "Vulcanization" in: "Science and Technology", Eds: Mark, J.E., Erman, B. and Eirich, F.R., Academic Press Limited, London, UK (1994).

7. Krejsa, M. R. \& Köenig, J. L. - Rubb. Chem. And Technol., 66, p.376 (1993).

8. Coran, A.Y. - "Vulcanization" in: "Encyclopedia of Polymer Science and Engineering", Eds: Mark, H., Bikales, N.M., Overberger, C. and Menges, G., John Wiley and Sons, New York, USA (1989).

9. Varghese, H.; Bhagawan, S. S. \& Thomas, S. - J. Appl. Polym. Sci, 71, p.2335 (1999).

10. Cook, S. - Kauts. Gummi Kunsts., 52, p.350 (1999).

11. Joseph, R.; George, K. E.; Samad, E. A. A. \& Francis, D. J. - Die Angewandte Makromolekulare Chemie, 163, p.37 (1988).

12. Ghoneim, A. M. \& Ismail, M. N. - Polym.-Plast. Technol. Eng., 38, p.979 (1999).

13. Suma, N.; Joseph, R. \& George, K. E. - J. Appl. Polym. Sci, 49, p.549 (1993).

14. Mastromatteo, R. P.; Mitchell, J. M. \& Brett, T. J. Rubber Chem. Technol., 44, p.1065 (1971).

15. Woods, M. E. \& Davidson, J. A. - Rubber Chem. Technol., 49, p.112 (1976).

16. Setua, D.K.; Pandey, K.N.; Saxena, A.K. \& Mathur, G.N., J. Appl. Polym. Sci., 74, p.480(1999).
17. Radusch, J. H.; Lämmer, E.; Halle, E. S. \& Dudik, M. - Kauts. Gummi Kunsts., 46, p.703 (1993).

18.Mullins, L. - J. Polym. Sci., 19, p.225 (1956).

19. Duchacek, V. - J. Appl. Polym. Sci., 16, p.3245 (1972).

20. Konar, B. B. - J. Appl. Polym. Sci., 63, p.233 (1997).

21. Mathew, G.; Singh, R. P.; Lakshminarayanan, R. \& Thomas, S. - J. Appl. Polym. Sci., 61, p.2035 (1996).

22. Elvira, M. R.; Macías, A.; Oteo, J. L.; Royo, J. \& Rubio, J. - Die Angewandte Makromolekulare Chemie, 227, p.43 (1995).

23. Ding, R.; Leonov, A. Y. \& Coran, A. Y. - Rubber Chem. Technol., 69, p.81 (1996).

24. Chough, S. H. \& Chang, D. H. - J. Appl. Polym. Sci., 61, p.449 (1996).

25. Tripathy, A. R.; Gosh, M. K. \& Das, C. K. - Kauts. Gummi Kunsts., 45, p.626 (1992).

26. Zapp, R. L. - Rubber Chem. Technol., 46, p.251 (1973).

27. Liau, W. B. \& Cheng, K. C. - Polymer, 39, p.6007 (1998).

28. Mason, P. - Polymer, 5, p.625 (1964).

Recebido: 25/09/01

Aprovado: 14/01/02 